\title{
NADH-dependent biosensor in Saccharomyces cerevisiae: principle and validation at the single cell level
}

\author{
Jan Dines Knudsen, Magnus Carlquist and Marie Gorwa-Grauslund
}

\begin{abstract}
A reporter system was constructed to measure perturbations in the $\mathrm{NADH} / \mathrm{NAD}^{+}$co-factor balance in yeast, by using the green fluorescent protein gene under the control of the GPD2 promoter that is induced under conditions of excess of NADH. High fluorescence levels were obtained in a glycerol 3-phosphate dehydrogenase double deletion strain (gpd14gpd2A), which is deficient in the ability to regenerate NAD ${ }^{+}$via glycerol formation. The responsiveness of the reporter system to externally induced perturbations in NADH oxidation was also evaluated in the gpd14gpd2 strain background by addition of acetoin, as well as by introduction of a set of heterologous xylose reductases (XRs) having different selectivities for NADH. Addition of acetoin during cell proliferation under oxygen-limited conditions resulted in a more than 2-fold decrease in mean fluorescence intensity as compared to the control experiment. Strains carrying XRs with different selectivities for NADH could be distinguished at the single cell level, so that the XR with the highest selectivity for NADH displayed the lowest fluorescence. In conclusion, the designed system successfully allowed for monitoring perturbations in the cellular redox metabolism caused by environmental changes, or by heterologous gene expression. The reporter system displayed high resolution in distinguishing cytosolic NADH oxidation capacity and hence has potential to be used for high-throughput screening based on the fluorescence of single cells.
\end{abstract}

Keywords: Saccharomyces cerevisiae; Redox balance; NADH biosensor; Single cell analysis

\section{Introduction}

The relative concentrations of $\mathrm{NADH} / \mathrm{NAD}^{+}$redox couple determine the thermodynamic feasibility of more than 100 reactions in cellular metabolism which places it at the core of the metabolic network of every organism including Saccharomyces cerevisiae (Förster et al. 2003). Efficient tools to measure the impact of redox enzymes or environmental perturbations on the cellular NADH levels are therefore needed (Murray et al. 2011). However quantification of redox couples in cells is complicated by several factors: the NADH and $\mathrm{NAD}^{+}$are compartmentalized in S. cerevisiae and the cofactors are to a large extent in a protein bound form (Veech et al. 1969; Bücher et al. 1972). Fluorescence imaging enables the distinction between free and bound redox couples, but not between NADH and NADPH (Zhang et al. 2002). In mammalian cells, coupled enzymatic reactions have

\footnotetext{
* Correspondence: marie-francoise.gorwa@tmb.lth.se

Division of Applied Microbiology, Department of Chemistry, Lund University, PO Box 124, SE 22100 Lund, Sweden
}

been used to determine the ratio between the cofactors NADH/NAD ${ }^{+}$(Williamson et al. 1967; Theobald et al. 1997). A similar approach has been applied for S. cerevisiae where mannitol-1-phosphate 5-dehydrogenase (M1PDH) was introduced and the cytosolic free $\mathrm{NADH} / \mathrm{NAD}^{+}$ratio was determined by the ratio between fructose-6-phosphate (F6P) and mannitol-1-phosphate (M1P) and the equilibrium constant for the reaction $\mathrm{M} 1 \mathrm{P}+\mathrm{NAD} \leftrightarrow \mathrm{F} 6 \mathrm{P}+$ $\mathrm{NADH}+\mathrm{H}^{+}$(Canelas et al. 2008). The approaches are however often tedious and not applicable for highthroughput screening.

In $S$. cerevisiae, the cytosolic enzyme glycerol-3phosphate dehydrogenase (GPD) 2 encoded by the GPD2 gene plays a central role in redox metabolism. Under anaerobiosis, GPD2 expression increases as the need for cytosolic reoxidation of NADH increases because Gpd2p acts as redox sink via the NADH-coupled reduction of dihydroxyacetone phosphate (DHAP) to glycerol 3-phosphate (G3P). G3P is further converted to 
glycerol (Eriksson et al. 1995; Ansell et al. 1997). The need for NADH reoxidation is thought to be linked, indirectly or indirectly, to the $\mathrm{NADH} / \mathrm{NAD}^{+}$ratio (Ansell et al. 1997). Glycerol production can also be initiated by the isoenzyme Gpd1p encoded by GPD1. However Gpd1p and Gpd2p are expressed under different growth conditions, as illustrated by their different cellular localization (Valadi et al. 2004). The physiological role of Gpd1p is also different from that Gpd2p as GPD1 is induced under osmotic stress (Blomberg and Adler 1989). Although the genes can complement each other's functions, a double deletion renders the yeast cell osmosensitive as well as incapable of anaerobic growth, due to the inability to reoxidize cytosolic NADH arising from biomass formation (van Dijken and Scheffers 1986; Nissen et al. 1997).

We used this knowledge to design a GFP-based biosensor for cytosolic NADH/NAD ${ }^{+}$levels by coupling the GPD2 promoter (GPD2p) to the $y E G F P 3$ gene encoding yeast enhanced green fluorescent protein, an established yeast reporter of the GFP class 2 (Cormack et al. 1996; Cormack et al. 1997; Zacharias and Tsien 2005). The applicability of fluorescent proteins as reporters has previously been demonstrated through the measurement of various user-specified cell properties. For example, GFP has been used for protein localization (Valadi et al. 2004), intracellular pH measurement (Valkonen et al. 2013), for monitoring cell growth mode and cell membrane robustness (Carlquist et al. 2012). Dardalhon et al. (2012) also implemented a fluorescent glutathione redox reporter in $S$. cerevisiae using a yellow fluorescent protein sensor that reports on the nuclear and cytosolic glutathione redox state i.e the glutathione/glutathione disulfide (GSH/GSSG) state, which is NADPH dependent.

The functionality of the $\mathrm{NADH} / \mathrm{NAD}^{+}$-ratio biosensor developed in this work was first evaluated by introducing it into $S$. cerevisiae strains with reduced NADH reoxidation capacity due to the deletion of Gpd1,2p encoding genes. The biosensor potential was then validated by evaluating the impact on cell fluorescence of acetoin addition and of the introduction of xylose reductases with different selectivities for $\mathrm{NADH}$.

\section{Methods}

\section{Strains}

$S$. cerevisiae strains used in the study are summarized in Table 1. Escherichia coli NEB 5 $\alpha$ (New England BioLabs, Ipswich, MA, USA) was used for subcloning. All strains were stored in $20 \%$ glycerol at $-80^{\circ} \mathrm{C}$. Yeast cells from freshly streaked YNB plates $(6.7 \mathrm{~g} / \mathrm{L}$ yeast nitrogen base without amino acids, $20 \mathrm{~g} / \mathrm{L}$ glucose, $2 \%$ agar) were used for all cultivations.
Table 1 Plasmids and yeast strains used in the present study

\begin{tabular}{|c|c|c|}
\hline $\begin{array}{l}\text { Strains and } \\
\text { plasmids }\end{array}$ & Relevant features & Reference \\
\hline \multicolumn{3}{|l|}{ Plasmids } \\
\hline Ylplac211 & URA3 & (Gietz and Sugino 1988) \\
\hline p424 & TRP1 Mumberg vector & (Mumberg et al. 1995) \\
\hline P425 & LEU2 Mumberg vector & (Mumberg et al. 1995) \\
\hline YEplacHXT & URA3 & (Karhumaa et al. 2005) \\
\hline pYGFP3 & URA3 ADH1p-yEGFP3-ADH1t & (Cormack et al. 1997) \\
\hline YlpJK01 & HIS3 GPD2p-yEGFP3-PGK1t & This study \\
\hline YEpJK01 & URA3 GPD2p-yEGFP3-PGK1t & This study \\
\hline YlpOB8 & $\begin{array}{l}\text { URA3 TDH3p-XYL1-ADH1t } \\
\text { PGK1p-XYL2-PGK1t }\end{array}$ & (Bengtsson et al. 2009) \\
\hline YlpDR7 & $\begin{array}{l}\text { URA3 TDH3p-XYL1(N272D)- } \\
\text { ADH1t PGK1p-XYL2-PGK1t }\end{array}$ & (Runquist et al. 2010) \\
\hline YlpCR01 & URA3 TDH3p-XYL1-ADH1t & This study \\
\hline YlpCR03 & $\begin{array}{l}\text { URA3 TDH3p-XYL1 } \\
\text { (N272D)-ADH1t }\end{array}$ & This study \\
\hline \multicolumn{3}{|c|}{ S. cerevisiae strains } \\
\hline CEN.PK2-1C & $\begin{array}{l}\text { MATa ura3-52 trp1-289 leu2-3, } \\
112 \text { his3 } 1 \text { MAL2-8C SUC2 }\end{array}$ & EUROSCARF \\
\hline CEN.PK113-7D & MATa, MAL2-8C SUC2 & EUROSCARF \\
\hline TMB4120 & $\begin{array}{l}\text { CEN.PK2-1C YEpJKO1 trp1:: } \\
\text { TRP1 leu2::LEU2 his3::HIS3 }\end{array}$ & This study \\
\hline TMB4121 & $\begin{array}{l}\text { CEN.PK2-1C YEpJK01 gpd1:: } \\
\text { TRP1 leu2::LEU2 his3::HIS3 }\end{array}$ & This study \\
\hline TMB4122 & $\begin{array}{l}\text { CEN.PK2-1C YEpJK01 trp1:: } \\
\text { TRP1 gpd2::LEU2 his3::HIS3 }\end{array}$ & This study \\
\hline TMB4123 & $\begin{array}{l}\text { CEN.PK2-1C YEPJK01 gpd1::: } \\
\text { TRP1 gpd2::LEU2 his3::HIS3 }\end{array}$ & This study \\
\hline TMB4132 & $\begin{array}{l}\text { CEN.PK2-1C his3::YipJK01 } \\
\text { trp1::TRP1 leu2::LEU2 ura3 }\end{array}$ & This study \\
\hline TMB4133 & $\begin{array}{l}\text { CEN.PK2-1C his3::YipJK01 } \\
\text { gpd1:::TRP1 gpd2:LEU2 ura3- }\end{array}$ & This study \\
\hline TMB4140 & TMB4132 ura3::Ylplac211 & This study \\
\hline TMB4141 & TMB4132 ura3::YlpCR01 & This study \\
\hline TMB4143 & TMB4132 ura3::YlpCR03 & This study \\
\hline TMB4144 & TMB4133 ura3::Ylplac211 & This study \\
\hline TMB4145 & TMB4133 ura3::YlpCR01 & This study \\
\hline TMB4147 & TMB4133 ura3::YIpCR03 & This study \\
\hline
\end{tabular}

\section{Molecular biology methods}

Plasmid DNA was prepared using the GeneJET Plasmid Miniprep Kit (Thermo Scientific, USA). Restriction and modification enzymes as well as T4 DNA ligase were obtained from the same manufacturer. The QIAquick gel extraction kit (QIagen, Hilden, Germany) was used for DNA extractions from agarose. All nucleotides were ordered at Eurofins (Germany). All genetic constructs were 
checked by sequencing (Eurofins, Germany). The primers used in the study are listed in Table 2.

Competent cells of $E$. coli NEB $5 \alpha$ were prepared and transformed by the method of Inoue et al. (1990) and yeast transformations were performed using the lithium acetate method (Gietz and Woods 2002). The cells were streaked out on selective medium afterwards. E. coli transformants were selected on Lysogene Broth (LB) plates (Sambrock and Russell 2001) with $50 \mu \mathrm{g} / \mathrm{ml}$ ampicillin
(IBI Shelton Scientific, Shelton, USA). S. cerevisiae transformants were selected on YNB plates supplemented with the requested amino acids or base.

\section{Construction of deletion and insertion cassettes}

The GPD1 deletion cassette was constructed by joining three PCR fragments through overlap extension PCR (Sandström et al. 2012). A nucleotide fragment homologous to the upstream region of GPD1 was PCR amplified

Table 2 Primers used in the present study

\begin{tabular}{|c|c|c|}
\hline Name & Description & Sequence \\
\hline TRP1_gpd1Over_f & $\begin{array}{l}\text { Forward primer for amplification of TRP1 (p424 as template) } \\
\text { with GPD1 overhangs }\end{array}$ & $\begin{array}{l}\text { TCCACAAACACAAATATTGATAATATAAAGAACGACATT } \\
\text { ACTATATATATAATATAG }\end{array}$ \\
\hline TRP1_gpd1Over_r & $\begin{array}{l}\text { Reverse primer for amplification of TRP1 (p424 as template) } \\
\text { with GPD1 overhangs }\end{array}$ & $\begin{array}{l}\text { AGTATGATATGTTATCTTCTCCAATAAATAGGCAAGTG } \\
\text { CACAAACAATAC }\end{array}$ \\
\hline \multirow[t]{2}{*}{ TRP1_gpd1_veri_f } & $\begin{array}{l}\text { Amplification of the } 5 \text { ' region of GPD1 (CEN.PK113-7D } \\
\text { as template) }\end{array}$ & TTCCATTCACATATCGTCTITGG \\
\hline & + Verification of GPD1 deletions & \\
\hline 5-OEGpd1_r & $\begin{array}{l}\text { Amplification of the } 5^{\prime} \text { region of GPD1 (CEN.PK113-7D } \\
\text { as template) }\end{array}$ & TATATTATCAATATTTGTGTTTGTGG \\
\hline 3-OEGpd1_f & $\begin{array}{l}\text { Amplification of the } 3 \text { ' region of GPD1 (CEN.PK113-7D } \\
\text { as template) }\end{array}$ & TTAATTGGAGAAAGATAACATATCATAC \\
\hline 3-OEGpd1_r & $\begin{array}{l}\text { Amplification of the } 3^{\prime} \text { region of GPD1 (CEN.PK113-7D } \\
\text { as template) }\end{array}$ & ATTTCTTAGGACGCCGCAAAATATC \\
\hline TRP1_gpd1_veri_r & Verification of GPD1 deletions & GAGGAACTCTTGGTATTCTTGCC \\
\hline LEU2_gpd2Over_f & $\begin{array}{l}\text { Forward primer for amplification of LEU2 (p425 as template) } \\
\text { with GPD2 overhangs }\end{array}$ & $\begin{array}{l}\text { TTCCTITCCTTCGCTCCCCTTCCTTATCATCGACTACG } \\
\text { TCGTAAGGCCG }\end{array}$ \\
\hline LEU2_gpd2Over_r & $\begin{array}{l}\text { Forward primer for amplification of LEU2 (p425 as template) } \\
\text { with GPD2 overhangs }\end{array}$ & $\begin{array}{l}\text { GATCAGAGGGGGAGGGGGGGGGAGAGTGTCGAGGAG } \\
\text { AACTTCTAGTATATC }\end{array}$ \\
\hline LEU2_gpd2OO_f & $\begin{array}{l}\text { Forward primer for nested PCR for adding homology regions } \\
\text { to the GPD2 deletion casette }\end{array}$ & $\begin{array}{l}\text { GTATTITGGTAGATTCAATTCTCTTTCCCTTTCCTITCCT } \\
\text { TCGCTCCC }\end{array}$ \\
\hline LEU2_gpd2OO_r & $\begin{array}{l}\text { Reverse primer for nested PCR for adding homology regions } \\
\text { to the GPD2 deletion casette }\end{array}$ & $\begin{array}{l}\text { AAATTGGTTGGGGGAAAAAGAGGCAACAGGAAAGATCA } \\
\text { GAGGGGGAGGG }\end{array}$ \\
\hline LEU2_gpd2_veri_f & Verification of GPD2 deletions & CGTGTATCTTCTAAGATTCAGTC \\
\hline LEU2_gpd2_veri_r & Verification of GPD2 deletions & CTAATGGCTCAACGTGATAAGG \\
\hline GPD2p_Xbal_r & Cloning of GPD2 from CEN.PK113-7D & CTTCTAGATTGATAAGGAAGGGGAG \\
\hline GPD2p_Sacl_f & Cloning of GPD2 from CEN.PK113-7D & TCGAGCTCCGCAATGTTTCGTTGG \\
\hline yEGFP_Xbal_f & Cloning of $y$ EGFP3 from pyEGFP3 & AATCTAGAAGCATTAAAAAATGTCTAAAGGTGAAG \\
\hline yEGFP_Pstl_r & Cloning of yEGFP3 from pyEGFP3 & TTCCTGCAGAGTTATTTGTACAATTCATCCATAC \\
\hline HIS3_flank_f & Cloning of HIS3 - Forward primer & CCAGGTATCGTITGAACACGG \\
\hline HIS3_flank_r & Cloning of HIS3 - Reverse primer & GCTCAGTTCAGCCATAATATG \\
\hline LEU2_flank_f & Cloning of LEU2 - Forward primer & GGATAATTATACTCTATTTCTCAAC \\
\hline LEU2_r & Cloning of LEU2 - Reverse primer & TTAAGCAAGGATTTCTTAAC \\
\hline TRP1_f & Cloning of TRP1 - Forward primer & ATGTCTGTTATTAATTTCAC \\
\hline TRP1_r & Cloning of TRP1-Reverse primer & CTATTTCTTAGCATTITTG \\
\hline Ylplac211_f & Cloning of GPD2p-yEGFP3-PGK1t & TTTATCTTCGTTTCCTGC \\
\hline Ylplac211_r & Cloning of GPD2p-yEGFP3-PGK1t & AAAACTGTATTATAAGTAAATG \\
\hline HIS3_O_f & $\begin{array}{l}\text { Cloning of HIS3- expression cassette, for change of } \\
\text { auxotrophic marker. }\end{array}$ & TTATAATACAGTITCCAGGTATCGTTGAACACGG \\
\hline HIS3_O_r & $\begin{array}{l}\text { Cloning of HIS3-expression cassette, for change of } \\
\text { auxotrophic marker. }\end{array}$ & GGAAACGAAGATAAATCGCTCAGTTCAGCCATAATATG \\
\hline
\end{tabular}


from genomic DNA of CEN.PK113-7D strain using the primer couple TRP1_gpd1_veri_f, 5-OEGpd1_r. The promoter, ORF and terminator of TRP1 were PCR amplified from a Mumberg p424 vector using the primers TRP1_gpd1Over_f, TRP1_gpd1Over_r. The downstream region of GPD1 was PCR amplified from genomic CEN. PK113-7D DNA using the primer couple 3-OEGpd1_f, 3-OEGpd1_r. The three fragments were all purified before being combined into one fragment using the nucleotides TRP1_gpd1Over_f, 3-OEGpd1_r.

The GPD2 deletion cassette was constructed by nested PCR on a PCR fragment containing the promoter, ORF and terminator of LEU2. The template fragment was constructed using the primers LEU2_gpd2Over_f, LEU2_gpd2Over_r. The fragment was purified and then used as template using the primers LEU2_gpd2OO_f, LEU2_gpd2OO_r. The added regions contained around $50 \mathrm{bp}$ regions homologous to the upstream and downstream region of GPD2, respectively.

Expression cassettes for curing tryptophan, leucine and histidine auxotrophies were constructed using CEN. PK113-7D as a template and corresponding primer couples (TRP1_f, TRP1_r, LEU2_flank_f, LEU2_r and HIS3_flank_f, HIS3_flank_r, respectively) to PCR amplify the promoter, gene and terminator of the respective genes.

\section{Plasmid construction}

Plasmid YEpJK01was constructed by ligation of three DNA fragments using T4 DNA ligase: 1) A $1.4 \mathrm{~kb}$ region directly upstream of the GPD2 ORF that was PCR amplified from CEN.PK113-7D genomic DNA using the primers GPD2p_XbaI_r and GPD2p_SacI_f, digested with $S a c \mathrm{I}$ and $\mathrm{Xba \textrm {I }}$ and purified, 2). The $y E G F P 3$ fragment that had been PCR amplified from pYGFP3, using the primers yEGFP_XbaI_f and yEGFP_PstI_r, digested with $\mathrm{XbaI}$ and PstI and finally purified, and 3) YEplacHXT digested with SacI and PstI. The ligation mix was used to transform E. coli.

Plasmid YIpJK01was constructed by excision of the GPD2p-yEGFP3-PGK1t cassette from YEpJK01 plasmid using the restriction enzymes $S p h \mathrm{I}$ and $S a c \mathrm{I}$. The fragment was purified from agarose gel and ligated to YIplac211 plasmid that was previously digested with the same restriction enzymes. The auxotrophic marker of the resulting plasmid was changed from URA3 to HIS3 by PCR amplifying the entire plasmid except for the URA3 region. The amplification was done using the primers YIplac211_f, YIplac211_r. The fragment was then recombined with a PCR fragment containing a HIS3 expression cassette using the In-Fusion ${ }^{\bullet}$ HD Cloning Kit. The HIS3 cassette was obtained from CEN.PK113-7D genomic DNA using the nucleotides HIS3_O_f, HIS3_O_r. The primers were designed according to the In-Fusion ${ }^{\circledR}$ HD Cloning Kit guidelines. The entire YEpJK01 plasmid was transformed into E. coli.
For the construction of YIpCRO1 and YIpCRO3, the $X R$-expression cassettes were excised from YIpOB8 and YIpDR7, respectively, using HindIII. The vector YIplac211 was cleaved using the same enzyme. The relevant fragments were isolated and purified. Ligation of YIplac211 and TDH3p-XYL1-ADH1t yielded YIpCR01. Ligation of YIplac211 and TDH3p-XYL1(N272D)-ADH1t yielded YIpCR03.

\section{Construction of yeast strains}

TMB4120, TMB4121, TMB4122, TMB4223 (Table 1) were all constructed using CEN.PK2-1C as parental strain. The strains were made prototrophic by transforming sequentially the CEN.PK2-1C strain with (i) the required expression cassettes for LEU2, TRP1 or HIS3, (ii) the GPD1 and/ or GPD2 deletion cassette and (iii) YEpJK01plasmid.

TMB4132 and TMB4133 were constructed by transformation of CEN.PK2-1C with expression cassettes for TRP1 and LEU2 or the GPD1 (TRP1) and GPD2 (LEU2) deletions cassettes. Finally the strains were transformed with YIpJK01 that was linearized with NheI.

TMB4140, TMB4141, TMB4143, TMB4144, TMB4145 and TMB4147 were constructed by transforming TMB4132 or TMB4133 with YIplac211, YIpCR01 or YIpCR03 respectively. All vectors were linearized with ApaI prior to transformation.

Single gene deletions were verified by PCR amplification whereas double deletions were verified by PCR screening and enzymatic assay (described below). GPD1 deletions were PCR verified using the nucleotide couple TRP1_gpd1_veri_f and TRP1_gpd1_veri_r. GPD2 deletions were verified using the nucleotide couples LEU2_gpd2_ veri_f and LEU2_gpd2_veri_r

\section{Cell cultivation}

For the initial Flourescence intensity (FI) measurements, single colony cells were taken from YNB plates not older than 2 weeks and inoculated in $250 \mathrm{ml}$ shake flasks containing $25 \mathrm{ml}$ YNB medium buffered to $\mathrm{pH} 5.5$ with potassium hydrogen phthalate and complemented with $2 \%$ glucose. The cells were grown overnight at $180 \mathrm{rpm}$ and $30^{\circ} \mathrm{C}$.

For the FI measurement of XR strains, pre-cultivations and cultivations were done in YNB medium buffered to pH 5.5 with potassium hydrogen phthalate and complemented with $2 \%$ galactose and $2 \%$ xylose. The cells from the pre-cultivation step were washed with sterile water before inoculation. Oxygen-limited cultures were performed in $65 \mathrm{~mL}$ flasks with $45 \mathrm{~mL}$ medium and ergosterol and Tween-80 were added to the medium at a final concentration of $0.42 \mathrm{~g} / \mathrm{L}$ and $0.01 \mathrm{~g} / \mathrm{L}$ respectively (Andreasen and Stier 1953, 1954). Bottles were closed with rubber stoppers and cannulas were used for gas outlet and sampling, thus minimizing oxygen transfer. Cultures were stirred gently with magnetic stirrers and 
incubated at $30^{\circ} \mathrm{C}$. Fermentation was performed in biological duplicates. Substrate consumption and product formation values were determined from samples withdrawn during $72 \mathrm{~h}$ of fermentation.

\section{Analyses}

Samples for $\mathrm{OD}_{600}$ were analyzed directly while samples for HPLC were kept at $-20^{\circ} \mathrm{C}$. Growth was monitored by measuring OD at $620 \mathrm{~nm}$.

Xylose, galactose, glycerol, ethanol and xylitol were separated and quantified by high-pressure liquid chromatography (HPLC) (Waters, Milford, MA, USA). The compounds were separated with a HPX-87P (Bio-Rad, Hercules, CA, USA) ion exchange column). Separation was performed at $80^{\circ} \mathrm{C}$, with $\mathrm{H}_{2} \mathrm{O}$ as mobile phase at a flow rate of $0.6 \mathrm{~mL} / \mathrm{min}$. A refractive index detector (RID6A; Shimadzu, Kyoto, Japan) was used for quantification.

A BD Accuri ${ }^{\mathrm{TM}}$ C6 (Becton-Dickinson, NJ, USA) was used for the evaluation of the redox biosensor in the different deletions strains. Fresh cells were used. Samples for flow cytometry were centrifuged for $1 \mathrm{~min}$ at $3000 \mathrm{~g}$ and $4^{\circ} \mathrm{C}$, and resuspended in saline solution. The impact of the reporter gene copy number on fluorescence heterogeneity was evaluated on A BD FACSort (Becton-Dickinson, NJ, USA) flow cytometer. Fresh cells were harvested at the late exponential phase. Evaluation of the biosensor responsiveness to redox perturbations by heterologous expression of NADH-dependent oxidoreductases was performed with a BD FACSAria III (Becton-Dickinson, NJ, USA) flow cytometer. Samples were frozen in phosphate buffer, $\mathrm{pH} 7$, $25 \%$ glycerol at $-80^{\circ} \mathrm{C}$ before being thawed and analyzed. Events were recorded with a rate of approximately 1,000 events per second. Excitation wavelength for the lasers used was $488 \mathrm{~nm}$. Fluorescence emission levels were measured using a band pass filter at 530/30 nm (FI). Processing and analysis of flow cytometry raw data was performed by using MatLab ${ }^{\circ}$ R2010b (The MathWorks, Inc., Natick, MA, USA). The measurement files, exported as fcs files by the flow cytometer FACSAria III, were imported into MATLAB, using a "fcs data reader" routine (by L. Balkay, University of Debrecen, Hungary), available on MATLAB W File Exchange website.

\section{Results}

\section{Construction and evaluation of the redox biosensor}

In order to construct a biosensor that responds to cytosolic $\mathrm{NADH} / \mathrm{NAD}^{+}$ratio, a $1.4 \mathrm{~kb}$ region upstream of the GPD2 open reading frame that includes the whole GPD2 promoter region (Eriksson et al. 1995), was cloned 18 bp upstream of the start codon of the $y E G F P 3$ gene (Cormack et al. 1997). The resulting GPD2p-yEGFP3 expression cassette was introduced in the multicopy vector YEplacHXT generating plasmid YEpJK01. The YEpJK01 plasmid was then introduced into a control laboratory strain as well as in redox engineered strains carrying either single or double deletion of GPD1 and GPD2 genes, thereby generating strains TMB4120 (control), TMB4121

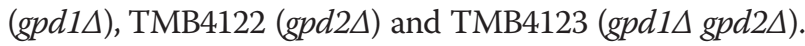

Strains were cultivated in batch mode under oxygenlimited conditions for 5 hours in defined mineral medium with glucose as sole carbon source, after which the FI was measured (Figure 1). At this time point, cells were in exponential growth phase and could be considered as being in pseudo-steady state with regards to regulation of

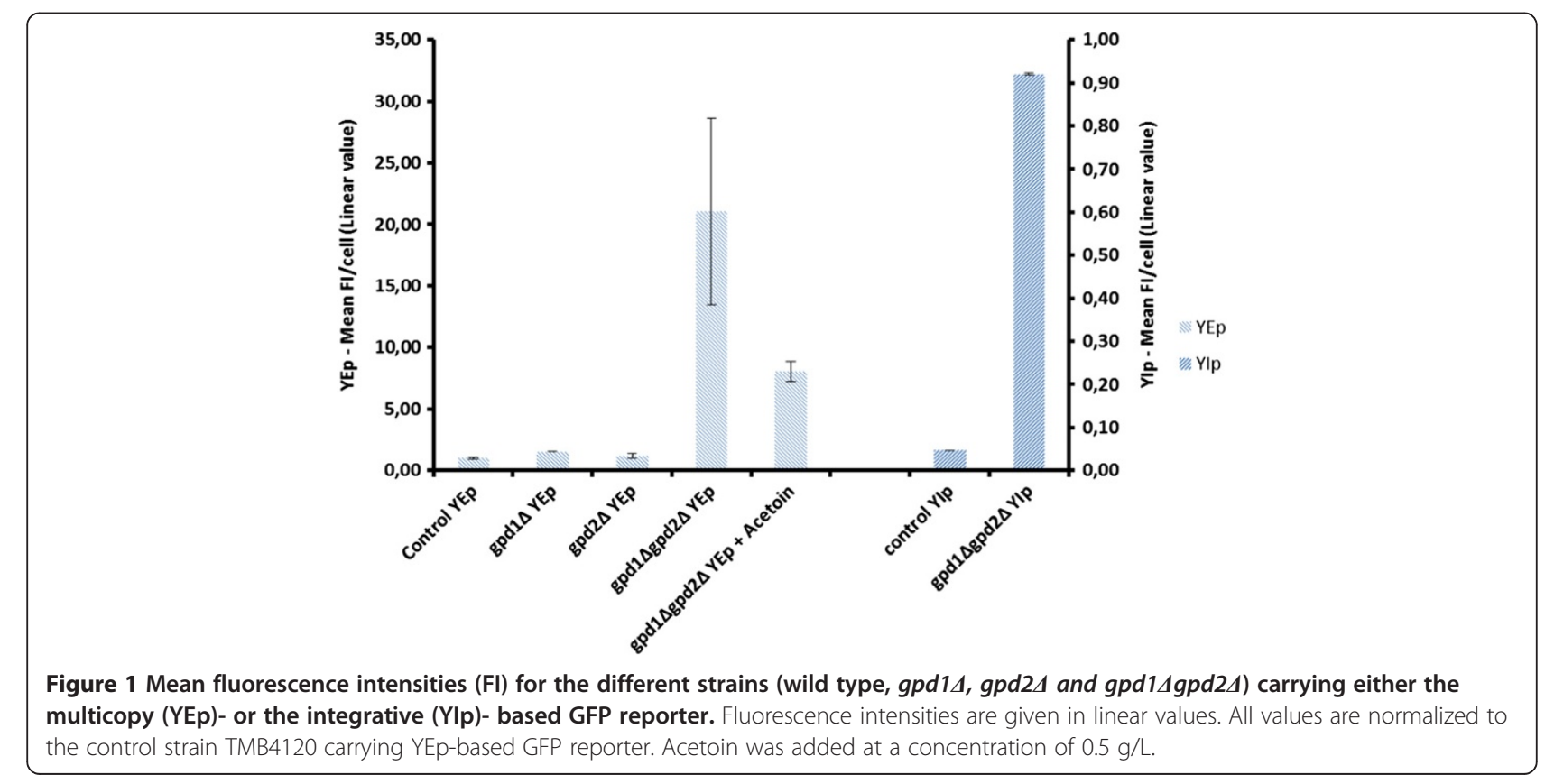


the glucose catabolic pathways. All redox engineered strain had higher FI than the control strain TMB4120. However the double deletion strain TMB4123 (gpd1A gpd2 $\Delta$ ) had the highest FI (21.5 fold higher than the control), whereas each single deletion had moderate increase in FI (1.5 and 1.2 fold for gpd1 $1 \Delta$ and gpd2A, respectively) (Figure 1). To further evaluate the biosensor responsiveness to perturbations in $\mathrm{NADH} / \mathrm{NAD}^{+}$ratio, the FI of TMB4123 (gpd1 $\operatorname{gpd} 2 \Delta)$ was measured after addition of acetoin (3-hydroxy-2-butanone) during respiro-fermentative growth under oxygen-limited conditions. Acetoin is an alternative redox sink to the glycerol shunt/pathway through its NADH-dependent conversion to 2,3-butanediol (Bruinenberg et al. 1983; Björkqvist et al. 1997). The reaction is catalyzed by a NADH-dependent 2,3-butanediol dehydrogenase encoded by $B D H 1$ (Gonzalez et al. 2000). Indeed the addition of acetoin led to reduced FI as the relative fluorescence decreased from 21.5 to 8.0 (Figure 1). Altogether our results demonstrated that the constructed biosensor reported on the cells capacity to oxidize NADH, and more generally on the $\mathrm{NAD}(\mathrm{H})$ cofactor balance.

\section{Impact of the reporter gene copy number on} fluorescence intensity and heterogeneity

To investigate the impact of the copy number of the reporter gene $y E G F P 3$ and only relate the expression level to the transcription step, the reporter cassette was also transferred to an integrative vector (YIp) and introduced into the same control and $g p d 1 \Delta \operatorname{gpd} 2 \Delta$ strain backgrounds, generating strains TMB4140 (control) and TMB4144 (gpd1 $\operatorname{gpd} 2 \Delta)$. Strains were cultivated in shake flasks with YNB media and 2\% glucose and FI was recorded. Similar trend was observed as for the YEp based reporter constructs. But the FI levels were lower, with strain TMB4140 (control- YIp) only emitting 4\% of the FI signal of TMB4120 (control- YEp). However, a clear difference in FI was again observed between the wild type and the

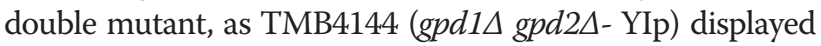
21.5 fold higher FI than its corresponding control TMB 4140 (Figure 1). Also the heterogeneity of the fluorescence signal that can be measured by the standard deviation of the population was lower in the YIp system (Figure 2). Standard deviations of 44 was measured in TMB 4144

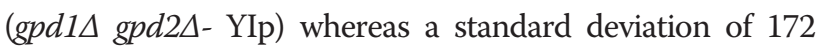
was obtained in TMB4123 (gpd1 $\operatorname{gpd} 2 \Delta$ - YEp). Thus, to reduce the influence of gene copy number and plasmid replication kinetics on FI output in the population, further experiments were performed using the YIp based sensor.

\section{Evaluation of the biosensor responsiveness to redox perturbations by heterologous expression of $\mathrm{NADH}$-dependent oxidoreductases}

The potential of the GPD2p-yEGFP3 redox biosensor to differentiate between reductases having different selectivities for NADH was also tested. For this purpose, two $\mathrm{NAD}(\mathrm{P}) \mathrm{H}$-dependent xylose reductases (XRs) that reduces

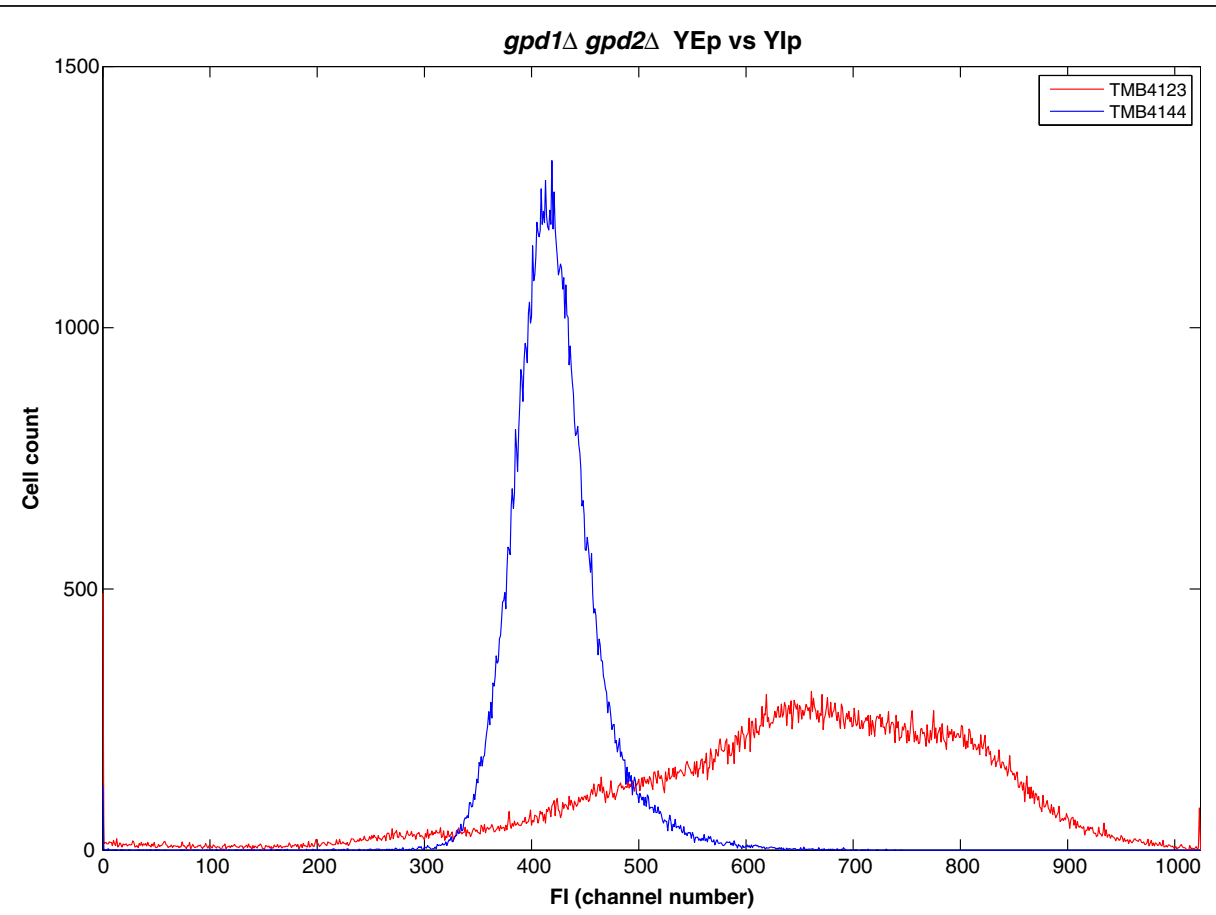

Figure 2 Histograms of the fluorescence intensity of strains TMB4123 (gpd14gpd2 YEp-GFP, "red dash") and TMB4144 (gpd1 4 gpd2 YIp-GFP, "blue dash") that were grown aerobically on glucose. 
xylose to xylitol using NADPH and NADH as cofactor were utilized: Pichia stipitis XR (wtXR) and the corresponding N272D mutant (mutXR) that display NADH/ NADPH selectivity ratios of 0.04 and 0.80 , respectively (Runquist et al. 2010). Both the non-engineered and the gpd1 $\operatorname{gpd} 2 \Delta$ yeast backgrounds were chosen as hosts as they represent the two redox extremes i.e. the strain with the best ability to reoxidize cytosolic NADH and the one with the poorest. The YIp-based reporter and XR constructs were introduced in these strains generating strains TMB4140 (control, no XR), TMB4141 (control, wtXR), TMB4143 (control, mutXR), TMB4144 (gpd1 1 gpd2A, no XR), TMB4145 (gpd1 1 gpd2 $\Delta$, wtXR) and TMB4147 (gpd1 1 gpd $2 \Delta$, mutXR).

Strains were cultivated under oxygen-limited conditions in sealed vials containing minimal medium with galactose as the carbon source and xylose as the substrate for the XR-catalysed reduction. Galactose was used because $S$. cerevisiae has a specific galactose uptake system, Gal2p (Cirillo 1968) that also transports xylose, and whose expression benefits xylose utilization (Johnston 1987; Hamacher et al. 2002). So, even though the yeast does not metabolize galactose as fast as glucose, the use of galactose allows the cell to have co-consumption of hexose and xylose (Bro et al. 2005; Garcia Sanchez et al. 2010). The growth profiles of the different strains are shown in Figures $3 \mathrm{~A}$ and B. During the first 24 hours, gpd $1 \Delta$ gpd $2 \Delta$ strains reached an OD of around 3, which was only slightly lower than the corresponding control strains. During the next 48 hours, however, TMB4147 strain (gpd1 $\operatorname{gpd} 2 \Delta$, mutXR) continued growing and reached an OD of 5.2 whereas strain TMB4145 (gpd1 $1 \Delta$ gpd2A, wtXR) only reached OD 3.7 while the OD of strain TMB4144 (gpd1A gpd2A, no XR) decreased to 2 (Figure 3B). Also TMB4147 strain carrying the XR with the highest selectivity for NADH (mutXR) almost

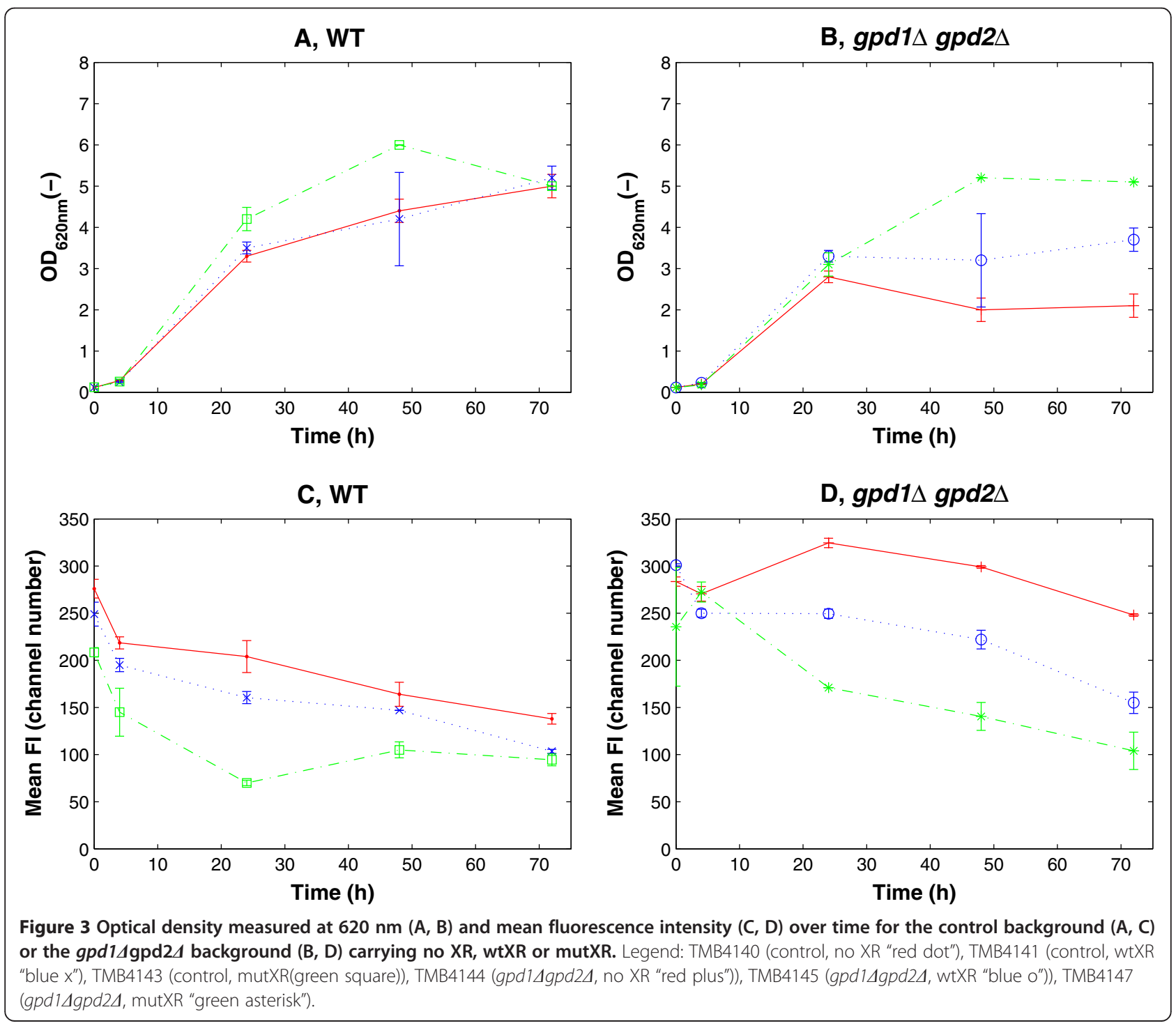


reached the same $\mathrm{OD}$ as the corresponding control strain TMB4143 (control, mutXR) (Figures 3A, B), which was not the case for the other strains carrying either no XR or wtXR. Improved NADH reoxidisation was expected to be coupled to the NADH-dependent xylose reduction to xylitol. Indeed high xylitol production, between 3 and $5 \mathrm{~g} / \mathrm{L}$, was measured during the first 48 hours of cultivations in all strains harboring a heterologous XR (Figure 4). Also the deletion strains produced xylitol faster, and in larger quantities than the control strains. Within the first 48 hours, there was no significant difference in xylitol production between strains carrying wtXR and mutXR, neither in the control strain background (Figure 4A) nor in the gpd1 $1 \Delta$ gpd2 $2 \Delta$ background (Figure 4B). However, the strains carrying mutXR reached slightly higher xylitol concentrations at the end of the cultivations. Xylitol was also produced in strains lacking heterologous XR, but to a significantly lower degree $(<0.8 \mathrm{~g} / \mathrm{L}$ after $70 \mathrm{~h})$. Xylitol may actually arise from the NADPH-dependent conversion of xylose by endogenous NADPH-dependent XR, such as the one encoded by GRE3 gene (Träff et al. 2002). In the case of XR-carrying strains, xylitol may also originate from the NADPH-catalysed reaction of the introduced XR. Glycerol production was observed for the double deletion strains carrying the control or mutated XR (Figure 4C, D), which may be explained by the fact that $P$. stipitis XR can use DHAP as substrate, which leads to glycerol formation (Jeppsson et al. 2003).

Both the control and the gpd1 $\operatorname{gpd} 2 \Delta$ strains showed the highest fluorescence at the beginning of the cultivation, followed by a gradual decrease until the end of fermentation. An inverse correlation was obtained between growth (Figure 3A, B) and the mean FIs (Figure 3C, D),

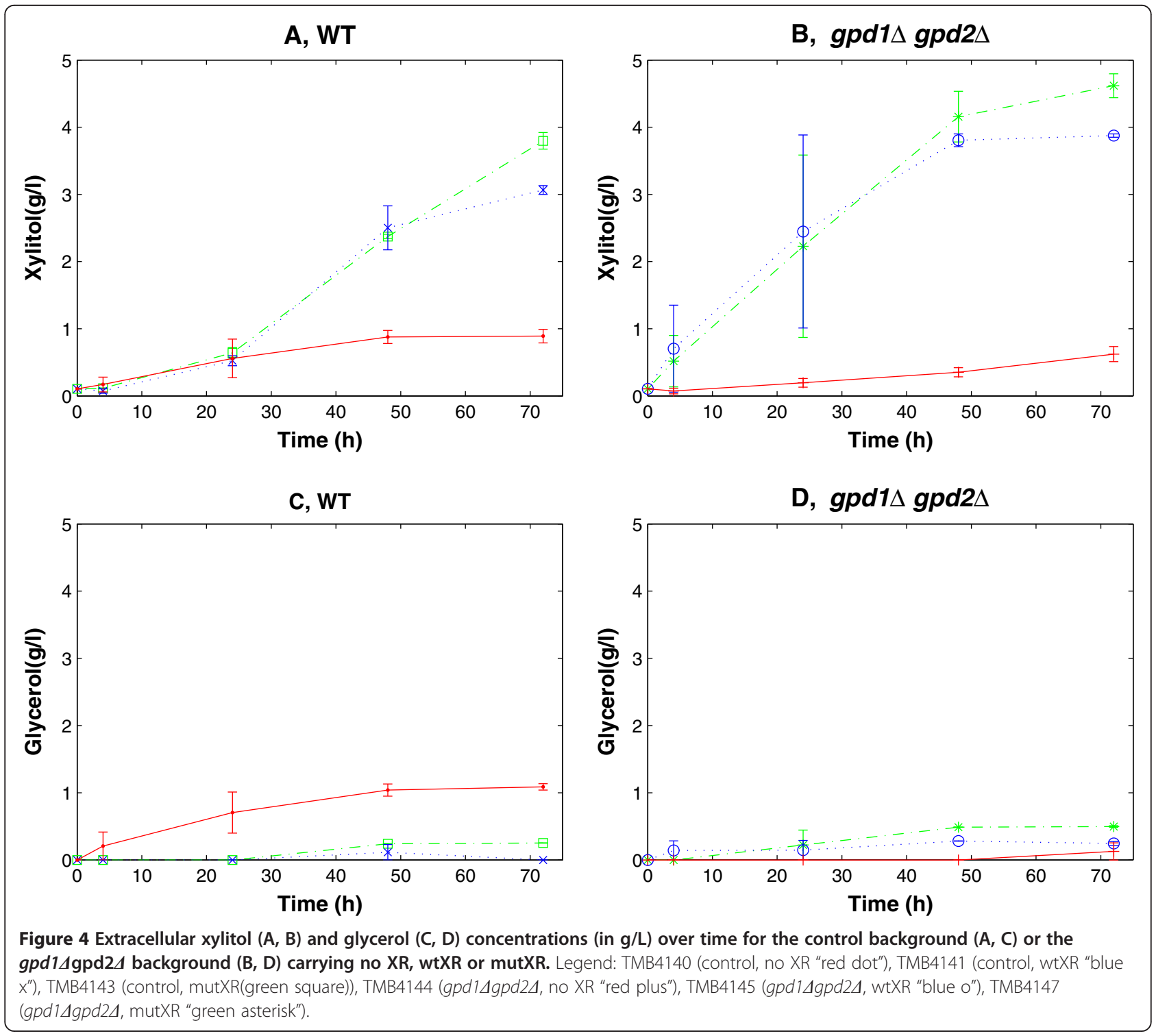


with the best growing strains having the lowest fluorescence both in the control and the gpd1 $\operatorname{gpd} 2 \Delta$ backgrounds. The difference in fluorescence was however less pronounced between the control strains than between the gpd $1 \Delta$ gpd $2 \Delta$ strains, which can be explained by the limited redox imbalance in the control strains.

The population histogram, i.e. the amount of single cells displaying a given FI, also clearly showed differences between the strains (Figures 5A, B). After 24 hours, the different populations displayed significantly different FIs, and were thus easily distinguished in an overlay plot. This was the case both in the control (Figure 5A) and in the gpd1 $1 \Delta$ gpd2 $\Delta$ (Figure 5B) strains, with mean FIs given in channel number units of 204 (control, no XR), 160 (control, wtXR), 70 (control, mutXR) or 325 (gpd1 $1 \Delta$ gpd2 $\Delta$, no XR), 250 (gpd1 $1 \Delta p d 2 \Delta$, wtXR) and 171 (gpd1 $\operatorname{god} 2 \Delta$, mutXR), respectively. Some overlap between strains was observed in both strain backgrounds, but it was more pronounced for the wild type strains than for the deletions strains

\section{Discussion}

Microbial populations exist in dynamic equilibrium with constantly changing conditions, which requires fast and fluid regulatory networks for optimizing cell functions. Among these, an accurate metabolic response is fundamental for the cell to maintain redox homeostasis. In $S$. cerevisiae, it is well known that a skewed ratio of cytosolic $\mathrm{NADH} / \mathrm{NAD}^{+}$can be compensated by glycerol production regulated by a fine-tuned expression of the GPD2 gene (Ansell et al. 1997; Björkqvist et al. 1997). This served as basis for the construction of our in vivo redox state reporter that enables green fluorescence to be used as an indicator of the cells ability to reoxidize $\mathrm{NADH}$, i.e. to the $\mathrm{NADH} / \mathrm{NAD}^{+}$ratio of the cell. This

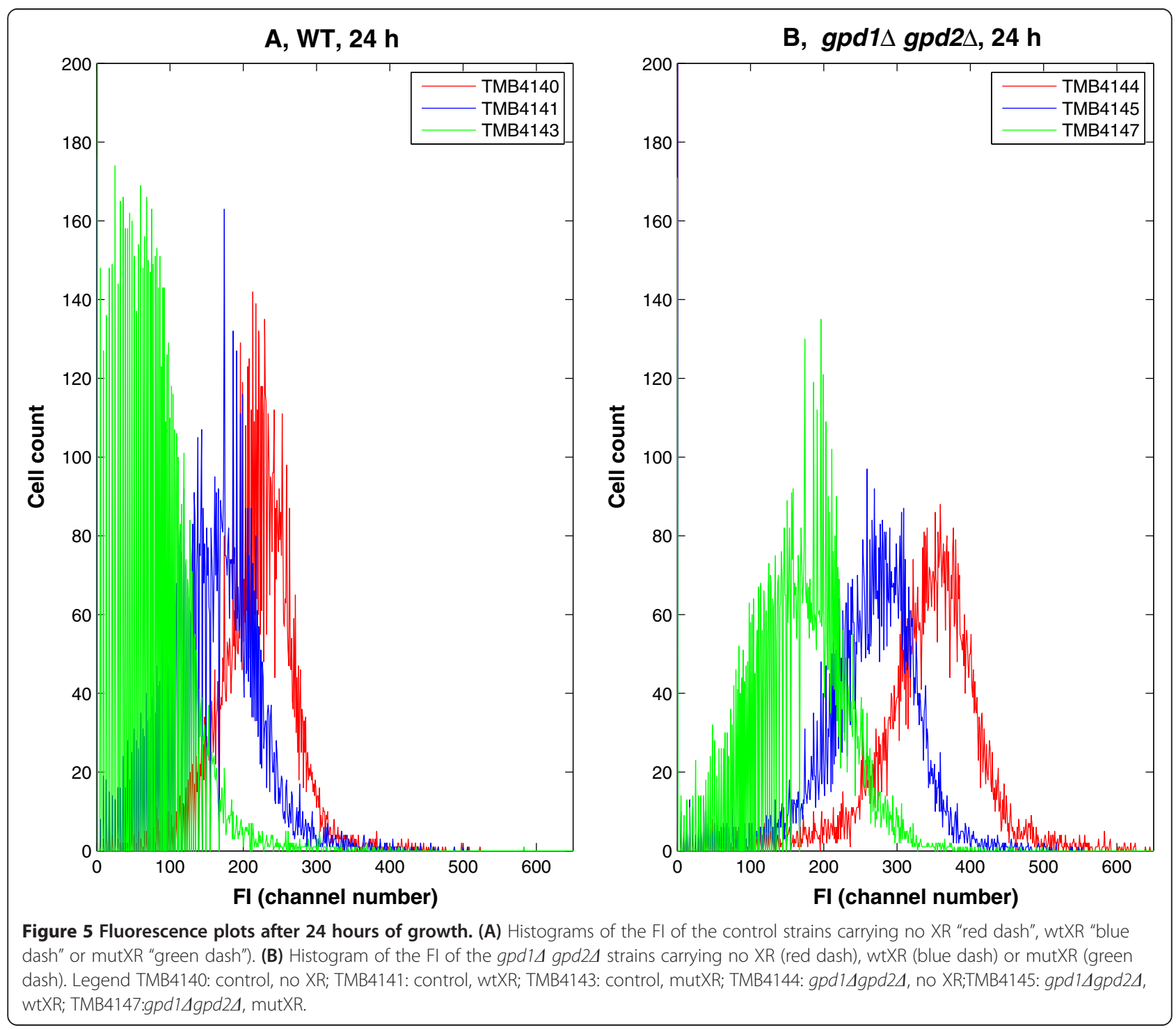


is, to our knowledge, the first biosensor to use the GPD2 promoter as sensor for the cytosolic NADH/NAD ${ }^{+}$cofactor balance.

By placing a GFP gene downstream of the GPD2 promoter, a direct relationship between green fluorescence intensity (FI) and glycerol formation or NADH accumulation was observed. In strains carrying double deletion of the GPD genes, the FI was significantly higher (21.5fold) than for the control strain, indicating a strong activation of the GPD2 promoter, and thereby verifying the lack of other enzymes to counteract the lacking NADH oxidation functionality. In parallel, FIs were in the same order of magnitude in single GPD deletion strain and in the control strain, also confirming the previously observed compensatory pattern between Gpdp isoenzymes in order to maintain $\mathrm{NAD}^{+}$regeneration via glycerol production (Ansell et al. 1997). The FI signal of the gpd $1 \Delta$ strain was also slightly higher than the FI of the $\operatorname{gpd} 2 \Delta$ strain and the control strain: $25 \%$ and 50\%, respectively, as expression of the GDP2, gene whose promoter is used in the constructed system, should be higher in the gpd1 1 strain, as a consequence of GPD2 compensating for the lacking GPD1 functionality. Finally the high responsiveness of the constructed reporter supported the hypothesis that GPD2 expression was directly connected to the cell need to oxidize NADH via glycerol formation. It cannot be excluded that FI may be correlated to more than the need for NADH oxidation via glycerol formation. Growth rate and the capacity to reoxidise NADH are closely related and thus FI will always be indirectly correlated to growth rate. As an example of this, the rescued growth rate of the gpd1 1 gpd $2 \Delta$ deletion strain by increased NADH oxidation led to significantly lower FI. However the oxygen concentration, which reflects the cells ability to replace the need for glycerol formation by aerobic respiration, was probably a more important parameter in that strain. Cultivating cells under oxygen-limited conditions could also have had implications for the FI measurements as fluorescent proteins require oxygen to mature. However, GFP expression in $S$. cerevisiae could previously be measured in cells that were cultivated under strict anaerobic conditions, most probably due to exposure to oxygen during the aerobic handling of samples. (Hebisch et al. 2013) previously demonstrated that the average maturation time in E. coli for GFP is around $6 \mathrm{~min}$, which is enough time for GFP to mature during the preparation for the FI measurement in the present study.

There was a clear difference between placing the reporter cassette on a multicopy plasmid or on a plasmid that gets integrated in the chromosome. Copy number per cell varies between 50 and 200 when using YEp vectors as opposed to one when using YIp vectors (Romanos et al. 1992). So placing the GFP-based reporter construct on
YEp plasmids led to a significantly higher FI than when using YIp-based construct. However, more defined fluorescence peaks were obtained when using the YIp system as opposed to the YEp system. This is in accordance with previous findings illustrating the stability issues of YEp-based systems compared to YIp-based systems (Ishii et al. 2009) and indicates lower degree of cell population heterogeneity in chromosomally integrated biosensors as compared to the YEp-based systems, which may later facilitate the distinction between any underlying subpopulations. Promoters can also have different levels of noise in the respective gene expression (Blake et al. 2003), which can lead to a higher heterogeneity of the signal. The low degree of heterogeneity in the YIp-based system further stresses the suitability of the GPD2 promoter for analysis of populations at the single cell level.

A 2-fold lowering of the GFP levels was observed when acetoin was added to GPD double deletion strains, which can be attributed to the induced NADH-dependent reduction of acetoin (Gonzalez et al. 2000). Acetoin or acetaldehyde, that is reduced to ethanol with the concurrent oxidation of $\mathrm{NADH}$, have previously been used to demonstrate the lack of alternative cytosolic reductive reactions in double deletion strain (Lidén et al. 1996; Ansell et al. 1997). In these studies, the involved oxidation reactions were efficient enough to recover sufficient redox balance for the cells to start growing. By monitoring FI instead, we wanted to investigate whether the constructed biosensor could monitor less drastic changes in the redox balance. To validate this hypothesis, we introduced XR enzymes with slightly different catalytic activity for NADH oxidation, namely two xylose reductases having NADH/ NADPH selectivity ratios of 0.04 and 0.80 respectively (Runquist et al. 2010). As expected, the biggest difference in growth was observed in the double deletion strains between the control and the strain carrying the mutXR, since more carbon was made available for growth when xylitol formation from xylose was used as redox sink instead of glycerol formation from glucose. This is also part of the explanation for the lower glycerol formation in the TMB4141 and TMB4143 as opposed to the TMB4140, as the cells can produce xylitol as a redox sink in addition to glycerol. A higher xylitol production could have been expected for the mutXR than wtXR in the gpd1Agpd2A strain, if only considering the NADH-dependent activity. However wtXR also has a significant activity with NADPH as a cofactor (Rizzi et al. 1988), which led to a non-negligible NADH-independent xylitol production. The maximum difference in OD was 2.5-fold after 48 hours of growth whereas the difference between the control strain and the strain carrying the wtXR was only 1.5 fold. So, in theory, the reductases could be distinguishable by repeating batch cultivations and using 
growth as selection. Assuming that after 48 hours one cell of TMB4144 (double deletion mutXR) grows to 2.5 times the cell density of the TMB4140 (double deletion no XR), it would take 27 batch cultivations of 48 hours each before one TMB4144 cell would outgrow $10^{9}$ TMB4140 cells by a factor of $1: 100$ (according to the following calculation: $\left.(1 / 2.5)^{27} \cdot 10^{9} \approx 0.01\right)$. Using fluorescence instead, the difference was clearly visible after a few hours. Increased xylitol formation and lower fluorescence were observed in strains carrying the wtXR and the mutXR. However, a clear difference in FIs was observed between the two strains whereas differences in xylitol levels were not that significant. The results, although qualitative, indicate that fluorescence was the more sensitive and faster method to discriminate between the two XR enzymes and their difference in NADHcatalyzed xylitol production and consequently their ability to affect the cytosolic $\mathrm{NADH} / \mathrm{NAD}^{+}$ratio. It also opens the possibility for high-throughput screening of enzymes with increased selectivity for NADH. In our cases, similar XR levels were measured (data not shown). However, in future screening, an enzyme with lower specificity for $\mathrm{NADH}$, but expressed at a higher level and giving a higher activity/per cell for NADH oxidation will lead to a lower FI. This limitation in the screening will, however, occur in any method based on the NADH pool and not only in the FI-based one. Another element that can be further considered when using a GFP-based reporter system for screening or analysis of the $\mathrm{NAD}(\mathrm{H})$ cofactor imbalance is the half-live of the chosen protein. The yEGFP3 protein used in the present study has been reported to have a half-life of about 7.5 hours (Mateus and Avery 2000), and indeed the intracellular GFP level did not respond rapidly to dynamic changes in external conditions. This implies that rapid changes in $\operatorname{NAD}(\mathrm{H})$ cofactor balancing may not be monitored with the developed system. However alternative GFP variants with shorter half-life, such as the GFP fused with a fragment of the CLN2 (yeast G1 cyclin) protein having a half-life of 30 minutes (Mateus and Avery 2000), could then be used instead.

Lately, much attention has been drawn in using FACS for screening large genetic space (Dietrich et al. 2010) and the technique has already been applied for the screening of NADPH-dependent alcohol dehydrogenases in E. coli (Siedler et al. 2014). However, the combination of a NADH biosensor and single-cell analysis has, to our knowledge, not yet been implemented in S. cerevisiae. Our tool offers the first single-cell high-throughput $\mathrm{NAD}(\mathrm{H})$ cofactor balance sensor in S. cerevisiae. In contrast to previously reported methods where extraction of intracellular $\mathrm{NAD}(\mathrm{H})$ metabolites was performed (Canelas et al. 2008), the method presented in this work is faster and allows for analysis with single-cell level resolution. Therefore the constructed tool offers possible applications in the selection of new NADH-dependent enzymes based on single cell sorting. Additionally it could be applied in monitoring the impact of various metabolic engineering strategies on the cytosolic $\mathrm{NAD}(\mathrm{H})$ cofactor balance of the cell.

\section{Competing interests}

The authors declare that they have no competing interests.

\section{Authors' contribution}

JDK, MC and MGG contributed equally in the design of the study. JDK performed the experimental work and wrote the manuscript. MC and MGG helped in drafting the manuscript. All authors have read and approved the submission of the manuscript.

\section{Acknowledgements}

This work was financially supported by the Swedish Research Council (Vetenskapsrådet). Camille Robert is acknowledged for assistance in creating some of the yeast strains. Stefan Morberg is acknowledged for technical assistance with the flow cytometry analysis. The pYGFP3 plasmid was kindly provided by Alistair Brown (University of Aberdeen).

Received: 26 August 2014 Accepted: 15 October 2014

Published online: 30 October 2014

\section{References}

Andreasen AA, Stier TJB (1953) Anaerobic nutrition of Saccharomyces cerevisiae. I. Ergosterol requirement for growth in a defined medium. J Cell Physiol 41(1):23-36

Andreasen AA, Stier TJB (1954) Anaerobic nutrition of Saccharomyces cerevisiae. II. Unsaturated fatty and requirement for growth in a defined medium. J Cell Physiol 43(3):271-281

Ansell R, Granath K, Hohmann S, Thevelein JM, Adler L (1997) The two isoenzymes for yeast $\mathrm{NAD}^{+}$-dependent glycerol 3-phosphate dehydrogenase encoded by GPD1 and GPD2 have distinct roles in osmoadaptation and redox regulation. EMBO J 16(9):2179-2187

Bengtsson O, Hahn-Hägerdal B, Gorwa-Grauslund MF (2009) Xylose reductase from Pichia stipitis with altered coenzyme preference improves ethanolic xylose fermentation by recombinant Saccharomyces cerevisiae. Biotechnol Biofuels 2:9

Björkqvist S, Ansell R, Adler L, Lidén G (1997) Physiological response to anaerobicity of glycerol-3-phosphate dehydrogenase mutants of Saccharomyces cerevisiae. Appl Environ Microbiol 63(1):128-132

Blake WJ MK, Cantor CR, Collins JJ (2003) Noise in eukaryotic gene expression. Nature 422(6932):633-637

Blomberg A, Adler L (1989) Roles of glycerol and glycerol-3-phosphate dehydrogenase $\left(\mathrm{NAD}^{+}\right)$in acquired osmotolerance of Saccharomyces cerevisiae. J Bacteriol 171(2):1087-1092

Bro C, Knudsen S, Regenberg B, Olsson L, Nielsen J (2005) Improvement of galactose uptake in Saccharomyces cerevisiae through overexpression of phosphoglucomutase: example of transcript analysis as a tool in inverse metabolic engineering. Appl Environ Microbiol 71(11):6465-6472

Bruinenberg P, de Bot PM, van Dijken J, Scheffers WA (1983) The role of redox balances in the anaerobic fermentation of xylose by yeasts. Eur J Appl Microbiol Biotechnol 18(5):287-292

Bücher T, Brauser B, Conze A, Klein F, Langguth O, Sies H (1972) State of oxidation-reduction and state of binding in the cytosolic NADH-system as disclosed by equilibration with extracellular lactate-pyruvate in hemoglobinfree perfused rat liver. Eur J Biochem 27(2):301-317

Canelas AB, van Gulik WM, Heijnen JJ (2008) Determination of the cytosolic free NAD/NADH ratio in Saccharomyces cerevisiae under steady-state and highly dynamic conditions. Biotechnol Bioeng 100(4):734-743

Carlquist M, Fernandes RL, Helmark S, Heins AL, Lundin L, Sorensen SJ, Gernaey $\mathrm{KV}$, Lantz AE (2012) Physiological heterogeneities in microbial populations and implications for physical stress tolerance. Microb Cell Fact 11:94

Cirillo VP (1968) Galactose transport in Saccharomyces cerevisiae. I. Nonmetabolized sugars as substrates and inducers of the galactose transport system. J Bacteriol 95(5):1727-1731

Cormack BP, Valdivia RH, Falkow S (1996) FACS-optimized mutants of the green fluorescent protein (GFP). Gene 173(1):33-38 
Cormack BP, Bertram G, Egerton M, Gow NA, Falkow S, Brown AJ (1997) Yeast-enhanced green fluorescent protein (yEGFP): a reporter of gene expression in Candida albicans. Microbiology 143(Pt 2):303-311

Dardalhon M, Kumar C, Iraqui I, Vernis L, Kienda G, Banach-Latapy A, He T, Chanet R, Faye G, Outten CE, Huang ME (2012) Redox-sensitive YFP sensors monitor dynamic nuclear and cytosolic glutathione redox changes. Free Radic Biol Med 52(11-12):2254-2265

Dietrich JA, McKee AE, Keasling JD (2010) High-throughput metabolic engineering: advances in small-molecule screening and selection. Annu Rev Biochem 79:563-590

Eriksson P, Andre L, Ansell R, Blomberg A, Adler L (1995) Cloning and characterization of GPD2, a second gene encoding sn-glycerol 3-phosphate dehydrogenase (NAD+) in Saccharomyces cerevisiae, and its comparison with GPD1. Mol Microbiol 17(1):95-107

Förster J, Famili I, Fu P, Palsson BO, Nielsen J (2003) Genome-scale reconstruction of the Saccharomyces cerevisiae metabolic network. Genome Res 13(2):244-253

Garcia Sanchez R, Hahn-Hägerdal B, Gorwa-Grauslund MF (2010) Cross-reactions between engineered xylose and galactose pathways in recombinant Saccharomyces cerevisiae. Biotechnol Biofuels 3:19

Gietz RD, Sugino A (1988) New yeast-Escherichia coli shuttle vectors constructed with in vitro mutagenized yeast genes lacking six-base pair restriction sites. Gene 74(2):527-534

Gietz RD, Woods RA (2002) Transformation of yeast by lithium acetate/ single-stranded carrier DNA/polyethylene glycol method. Methods Enzymol 350:87-96

Gonzalez E, Fernandez MR, Larroy C, Sola L, Pericas MA, Pares X, Biosca JA (2000) Characterization of a (2R,3R)-2,3-butanediol dehydrogenase as the Saccharomyces cerevisiae YALO60W gene product. Disruption and induction of the gene. J Biol Chem 275(46):35876-35885

Hamacher T, Becker J, Gardonyi M, Hahn-Hägerdal B, Boles E (2002) Characterization of the xylose-transporting properties of yeast hexose transporters and their influence on xylose utilization. Microbiology 148(Pt 9):2783-2788

Hebisch E, Knebel J, Landsberg J, Frey E, Leisner M (2013) High variation of fluorescence protein maturation times in closely related Escherichia coli strains. PLoS One 8(10):e75991

Inoue H, Nojima H, Okayama H (1990) High efficiency transformation of Escherichia coli with plasmids. Gene 96(1):23-28

Ishii J, Izawa K, Matsumura S, Wakamura K, Tanino T, Tanaka T, Ogino C, Fukuda $\mathrm{H}$, Kondo A (2009) A simple and immediate method for simultaneously evaluating expression level and plasmid maintenance in yeast. J Biochem 145(6):701-708

Jeppsson M, Träff K, Johansson B, Hahn-Hägerdal B, Gorwa-Grauslund MF (2003) Effect of enhanced xylose reductase activity on xylose consumption and product distribution in xylose-fermenting recombinant Saccharomyces cerevisiae. FEMS Yeast Res 3(2):167-175

Johnston M (1987) A model fungal gene regulatory mechanism: the GAL genes of Saccharomyces cerevisiae. Microbiol Rev 51(4):458-476

Karhumaa K, Hahn-Hägerdal B, Gorwa-Grauslund MF (2005) Investigation of limiting metabolic steps in the utilization of xylose by recombinant Saccharomyces cerevisiae using metabolic engineering. Yeast 22(5):359-368

Lidén G, Walfridsson M, Ansell R, Anderlund M, Adler L, Hahn-Hägerdal B (1996) A glycerol-3-phosphate dehydrogenase-deficient mutant of Saccharomyces cerevisiae expressing the heterologous XYL1 gene. Appl Environ Microbiol 62(10):3894-3896

Mateus C, Avery SV (2000) Destabilized green fluorescent protein for monitoring dynamic changes in yeast gene expression with flow cytometry. Yeast 16(14):1313-1323

Mumberg D, Müller R, Funk M (1995) Yeast vectors for the controlled expression of heterologous proteins in different genetic backgrounds. Gene 156(1):119-122

Murray DB, Haynes K, Tomita M (2011) Redox regulation in respiring Saccharomyces cerevisiae. Biochim Biophys Acta 1810(10):945-958

Nissen TL, Schulze U, Nielsen J, Villadsen J (1997) Flux distributions in anaerobic glucose-limited continuous cultures of Saccharomyces cerevisiae. Microbiology 143(Pt 1):203-218

Rizzi M, Erlemann P, Bui-Thanh N-A, Dellweg H (1988) Xylose fermentation by yeasts. Appl Microbiol Biotechnol 29(2-3):148-154

Romanos MA, Scorer CA, Clare JJ (1992) Foreign gene expression in yeast: a review. Yeast 8(6):423-488

Runquist D, Hahn-Hägerdal B, Bettiga M (2010) Increased ethanol productivity in xylose-utilizing Saccharomyces cerevisiae via a randomly mutagenized xylose reductase. Appl Environ Microbiol 76(23):7796-7802
Sambrock J, Russell DW (2001) Molecular Cloning, a Laboratory Manual. Press Cold Spring Harbor Laboratory, New York, USA

Sandström AG, Wikmark Y, Engström K, Nyhlen J, Bäckvall JE (2012) Combinatorial reshaping of the Candida antarctica lipase $A$ substrate pocket for enantioselectivity using an extremely condensed library. Proc Natl Acad Sci U S A 109(1):78-83

Siedler S, Schendzielorz G, Binder S, Eggeling L, Bringer S, Bott M (2014) SoxR as a single-cell biosensor for NADPH-consuming enzymes in Escherichia coli. ACS Synth Biol 3(1):41-47

Theobald U, Mailinger W, Baltes M, Rizzi M, Reuss M (1997) In vivo analysis of metabolic dynamics in Saccharomyces cerevisiae : I. Experimental observations. Biotechnol Bioeng 55(2):305-316

Träff KL, Jönsson L, Hahn-Hägerdal B (2002) Putative xylose and arabinose reductases in Saccharomyces cerevisiae. Yeast 19(14):1233-1241

Valadi A, Granath K, Gustafsson L, Adler L (2004) Distinct intracellular localization of Gpd1p and Gpd2p, the two yeast isoforms of NAD+-dependent glycerol-3-phosphate dehydrogenase, explains their different contributions to redox-driven glycerol production. J Biol Chem 279(38):39677-39685

Valkonen M, Mojzita D, Penttila M, Bencina M (2013) Noninvasive high-throughput single-cell analysis of the intracellular $\mathrm{pH}$ of Saccharomyces cerevisiae by ratiometric flow cytometry. Appl Environ Microbiol 79(23):7179-7187

van Dijken JP, Scheffers WA (1986) Redox balances in the metabolism of sugars by yeasts. FEMS Microbiol Lett 32(3-4):199-224

Veech RL, Eggleston LV, Krebs HA (1969) The redox state of free nicotinamideadenine dinucleotide phosphate in the cytoplasm of rat liver. Biochem J 115(4):609-619

Williamson DH, Lund P, Krebs HA (1967) The redox state of free nicotinamide-adenine dinucleotide in the cytoplasm and mitochondria of rat liver. Biochem J 103(2):514-527

Zacharias DA, Tsien RY (2005) Molecular Biology and Mutation of Green Fluorescent Protein. Green Fluorescent Protein. John Wiley \& Sons, Inc., In, pp 83-120

Zhang Q, Piston DW, Goodman RH (2002) Regulation of corepressor function by nuclear NADH. Science 295(5561):1895-1897

doi:10.1186/s13568-014-0081-4

Cite this article as: Knudsen et al: NADH-dependent biosensor in

Saccharomyces cerevisiae: principle and validation at the single cell level. AMB Express 2014 4:81.

\section{Submit your manuscript to a SpringerOpen ${ }^{\odot}$ journal and benefit from:}

- Convenient online submission

- Rigorous peer review

- Immediate publication on acceptance

- Open access: articles freely available online

- High visibility within the field

- Retaining the copyright to your article

Submit your next manuscript at springeropen.com 